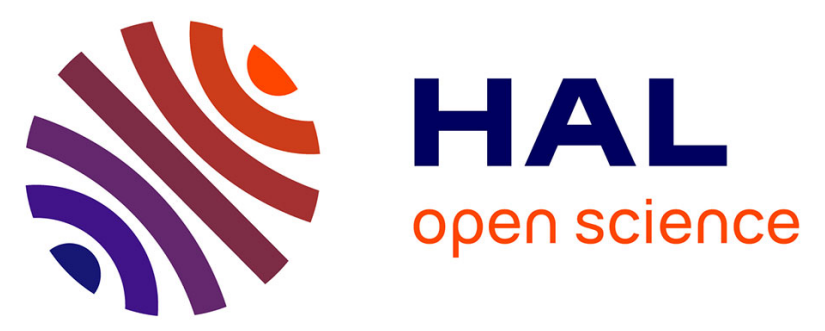

\title{
Low-Noise ASIC and New Layout of Multipolar Electrode for Both High ENG Selectivity and Parasitic Signal Rejection
}

\author{
Serge Bernard, Lionel Gouyet, Guy Cathébras, Fabien Soulier, David \\ Guiraud, Yves Bertrand
}

\section{To cite this version:}

Serge Bernard, Lionel Gouyet, Guy Cathébras, Fabien Soulier, David Guiraud, et al.. Low-Noise ASIC and New Layout of Multipolar Electrode for Both High ENG Selectivity and Parasitic Signal Rejection. ICECS'07: International Conference on Electronics, Circuits and Systems, Dec 2007, Marrakech, Morocco, Morocco. pp.A4L-A. lirmm-00195231

\section{HAL Id: lirmm-00195231 https://hal-lirmm.ccsd.cnrs.fr/lirmm-00195231}

Submitted on 10 Dec 2007

HAL is a multi-disciplinary open access archive for the deposit and dissemination of scientific research documents, whether they are published or not. The documents may come from teaching and research institutions in France or abroad, or from public or private research centers.
L'archive ouverte pluridisciplinaire $\mathbf{H A L}$, est destinée au dépôt et à la diffusion de documents scientifiques de niveau recherche, publiés ou non, émanant des établissements d'enseignement et de recherche français ou étrangers, des laboratoires publics ou privés. 


\title{
Low-noise ASIC and New Layout of Multipolar Electrode for both High ENG Selectivity and Parasitic Signal Rejection
}

\author{
Bernard S, Gouyet L, Cathébras G, Soulier F, Guiraud D and Bertrand Y \\ LIRMM, Université Montpellier II - CNRS - INRIA, 161 rue Ada, 34392 Montpellier, France \\ Email: lastnamedirmm.fr
}

\begin{abstract}
In order to extract and separate Action Potential (AP) signals according to their nerve fascicule origins, we propose a new architecture of a multipolar cuff electrode and an optimized integrated acquisition circuit. The proposed electrode has a specific layout of a large number of poles in order to both reject parasitic signals, such as electromyogram and provide a maximum of spatial selectivity for ENG signals. For one channel to be recorded, we need to consider seven recording sites. A low-noise integrated circuit (ASIC) has been designed in order to perform this first step of analog processing on each set of seven considered poles
\end{abstract}

\section{INTRODUCTION}

In a context of neural system pathologies such as spinal cord injury, Functional Electrical Stimulation (FES) techniques are the possible alternatives to restore lost sensory or motor abilities. These techniques consist in generating artificial contraction by electrical stimulation of nerve or muscle. In FES system a direct opened loop control doesn't allow efficient stimulation. In order to provide a loopback control we need sensory information (force, contact...) [1]. An attractive solution consists in using the natural sensors. The sensory information is propagated along associated afferent fibers. But unfortunately, in peripheral nerves the complete nerve activity due to the large number of axons makes the extraction of the studied signal particularly hard. Moreover the sensory signal seen throught the nerve, the Electroneurogram (ENG), is a very low amplitude signal compared with the amplitude of parasitic signals. For instance, on a monopolar recording, Electromyogram (EMG) created by muscle activity have amplitude about three orders of magnitude higher than the ENG. In this context, the two main objectives to be able to exploit natural sensors are:

- to find a solution to separate the useful information from the complete ENG signal;

- to reject the parasitic external signals.

The classical solution consists in using multipolar electrodes, but from tripole [2] to nine pole electrode [3], [4], the selectivity of the neural information is not efficient enough to be suitable in (a)

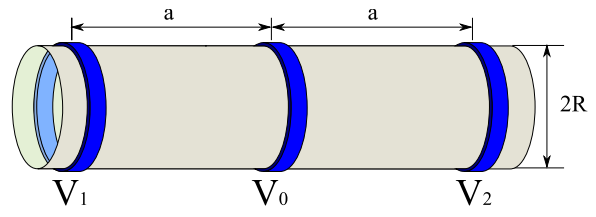

(b)

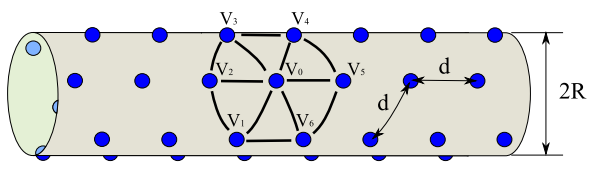

Figure 1. Tripolar cuff (a) and hexagonal electrode (b) models

closed loop FES system. In this paper, we consider a new configuration of the cuff electrode with a large number of poles laid-out in an hexagonal tessellation. Because of the very low level of studied signals we propose to perform the maximum of signal processing as close as possible to the nerve. Therefore, in this configuration, a group of seven poles can behave, with suitable low level analog signal processing, like a kind of a directive antenna. Moreover, the large number of poles will give enough channels in order to apply source separation signal processing on the ENG.

In section II we present the architecture of the proposed multipolar cuff electrode. The overview of the ASIC used for amplification of the ENG signal is given setion III. The section IV presents a set of simulation results with the proposed electrode based on a specific AP modeling. Finally, section $\mathrm{V}$ gives some concluding remarks and possible perspectives.

\section{ELECTRODE FOR ENG RECORDING}

Cuff electrodes have been the most used in the last ten years [5]-[7]. They are relatively easy to implant, they are not invasive for the nerve and implantation is very stable and thus allows chronic experiments. ENG can be recorded as the potential difference created on the electrodes by the charges associated to the action potentials (AP) propagating along the nerve fibers. Fig. 1-a shows a typical tripolar cuff electrode. When recording with this kind of electrode, a classic method to reject parasitical signals consists in calculating the 


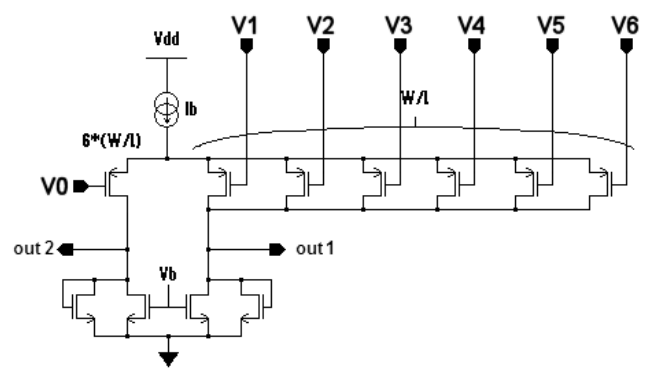

Figure 2. Seven input preamplifier

average of the potential differences between the central pole and each of the outer poles [8], [9]:

$$
V_{\text {rec }}=\frac{\left(V_{0}-V_{1}\right)+\left(V_{0}-V_{2}\right)}{2}=V_{0}-\frac{V_{1}+V_{2}}{2}
$$

With the aim of obtaining more localized measures, we propose to use a structure with a large number of poles in an hexagonal configuration (Fig. 1-b: 42 poles). Let us call this an hexagonal cuff. On this electrode, poles will be gathered in hexagonal patches. On each patch, we calculate the mean of the potential differences between the central pole and each of the peripheral poles:

$$
V_{\text {rec }}=\frac{1}{6} \sum_{i=1}^{6}\left(V_{0}-V_{i}\right)=V_{0}-\sum_{i=1}^{6} \frac{V_{i}}{6}
$$

\section{ENG AMPLIFIER}

The sensitivity of the sensor relies on the signal processing in order to compute correctly $V_{\text {rec }}$. For this purpose, we have designed a seven channels ASIC. Each channel compute a weighted difference between the measurement point and the six closest surrounding points. This is done in the analog domain using the preamplifier shown on figure 2. This preamplifier is build around a differential pair whose negative input transistor was split into six transistors (six times smaller, of course). It has a voltage gain that is about 100 and it is followed by an instrumentation amplifier whose gain is configurable between $6 \mathrm{~dB}$ and $80 \mathrm{~dB}$. Each channel is composed of one preamplifier followed by an instrumentation amplifier.

This circuit was designed to give an inputreferred noise below $1 \mu \mathrm{V}_{\text {rms }}$, a CMMR above $60 \mathrm{~dB}$ and a sufficient gain, i.e greater than $60 \mathrm{~dB}$ ; all these parameters in the bandwidth of interest $(1 \mathrm{~Hz} \leq f \leq 3 \mathrm{kHz})$. The performances expected for this amplifier are given in Table I (the noise is measured in the band $1 \mathrm{~Hz}-3 \mathrm{kHz}$ ).

A microphotography of the fabricated circuit is presented Fig. 3. This circuit was designed in CMOS AMS 0.35- $\mu \mathrm{m}$ technology.
Table I

AMPLIFIER CHARACTERISTICS (SIMULATION)

\begin{tabular}{|l|c|}
\hline Active area (7 channels) & $1.16 \mathrm{~mm}^{2}$ \\
\hline Supply voltage & $3.3 \mathrm{~V}$ \\
\hline DC Current (Preamp) & $20 \mu \mathrm{A}$ \\
\hline Voltage gain (Preamp) & $100(40 \mathrm{~dB})$ \\
\hline CMRR (Preamp) & $80 \mathrm{~dB}(10 \mathrm{kHz})$ \\
\hline Voltage gain (Inst amp) & $2 \leq G \leq 10000$ \\
\hline CMRR (Full amp) & $80 \mathrm{~dB}(10 \mathrm{kHz})$ \\
\hline Input-ref. noise (Preamp) & $0.672 \mu \mathrm{V} \mathrm{RMS}$ \\
\hline Input-ref. noise (Full amp) & $0.677 \mu \mathrm{V} \mathrm{RMS}$ \\
\hline Bandwidth (Full amp) & $76 \mathrm{kHz}$ \\
\hline
\end{tabular}

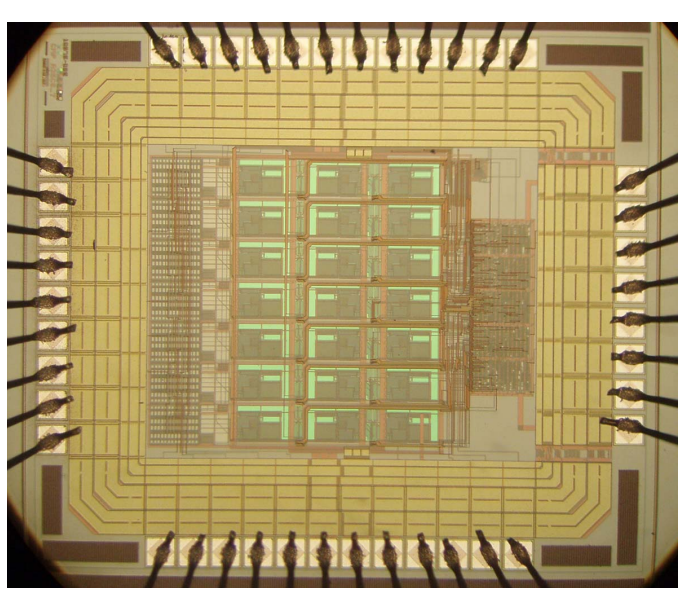

Figure 3. Microphotograph of the seven-channel prototype

\section{VALIDATION}

\section{A. Modeling}

In order to evaluate the performances of our system, we need a model for the extracellular electric field created by an action potential. Let us consider a $10 \mu \mathrm{m}$ diameter myelinated axon. Its Ranvier nodes are $1 \mu \mathrm{m}$ long, while their diameter is $6 \mu \mathrm{m}$ and their spacing is $1 \mathrm{~mm}$. Let us call $\Omega$ the centre of the Ranvier node. When the AP is present at this node, we can model it as a $6 \mu \mathrm{m}$ diameter circle, perpendicular to the axon axis, with a positive charge $+q$ at its centre $(\Omega)$ and a negative charge $-q$ spread on the circle. The potential created at a point $M$ of the space by this AP can be approximated by:

$$
V(M)=\frac{q a^{2}}{8 \pi \varepsilon_{0} \varepsilon_{r} r^{3}}\left(1-\frac{3}{2} \sin ^{2} \psi\right)
$$

In this expression, $a$ is the radius of the ranvier node $(3 \mu \mathrm{m}), r$ is the distance between $\Omega$ and $M$, while $\psi$ is the angle between the axe of the axon and $\overrightarrow{\Omega M}$. This approximation, valid for $r \gg a$, is in good accordance with measurements. In particular, we can see that $V(M)$ is negative for $\psi=\pi / 2$ [10, page 81]. 


\section{B. Simulation Setup}

The charge $q$ can be easily estimated from the characteristics of the Ranvier node. For this study, we took $q \simeq 20 \mathrm{fC}$ and $\varepsilon_{r} \simeq 80$.

The model given by equation 3 was used to evaluate the sensitivity of the electrodes to action potentials occurring inside the nerve. For the evaluation of the rejection of parasitic signals, we must first recall that EMG are also action potentials, creating the same kind of electric field. But, in this case, we cannot make any assumption on the value of $\psi$. So, to evaluate the external sensitivity of electrodes, we chosen to use only a $1 / r^{3}$ model, unable to give voltages, but sufficient to compare the sensitivities of various electrodes.

Given the position of a single AP we can easily calculate the induced potential on each pole of the hexagonal cuff, since they are very small. For the tripolar cuff, we need to average the potential on each ring. This lead to an elliptic integral we have solved using numerical methods.

In the following, we compare a tripolar cuff electrode, whose diameter is $2 R=3 \mathrm{~mm}$ and ring spacing is $a=4 R$, with one patch of our hexagonal cuff. To get comparable results, this hexagonal cuff has the same diameter $(2 R=3 \mathrm{~mm})$ and the spacing between poles is $d=R$. Since this patch is partially wrapped around the nerve, we considered also another patch perfectly flat.

For all the calculations, the coordinates were fixed as follows: the origin $O$ is at the centre of the cuff electrode. The $O x$ axis is the axis of the nerve (and, obviously, of the cuff). The $O y$ axis passes by the centre of the considered patch (which is perpendicular to this axe). Last the $O z$ axe is placed to form a direct trihedron with $O x$ and $O y$.

\section{Internal sensitivity}

Figure 4 shows the radial sensitivities of the three electrodes (tripolar cuff, planar hexagonal patch and wrapped hexagonal patch) that we compare. The vertical axis is the value of $V_{\text {rec }}($ in $\mathrm{dB} \mu \mathrm{V}$ ) calculated for an AP placed on the $O y$ axis, at abscissa $y R$. The graph shows clearly that while the sensitivity of the tripolar cuff is quasi constant on the section of the nerve, the sensitivity of the hexagonal patch is far higher (up to $30 \mathrm{~dB}$ ) when considering an AP located between the centre of the patch and the centre of the cuff.

Figure 5 shows the longitudinal sensitivities of the three considered electrodes. The AP is placed on a line, parallel to $O x$, cutting $O y$ at abscissa $0.8 R$. On this figure, we can see that the sensitivity of the tripolar is far lower than the sensitivity of any of the hexagonal patches.

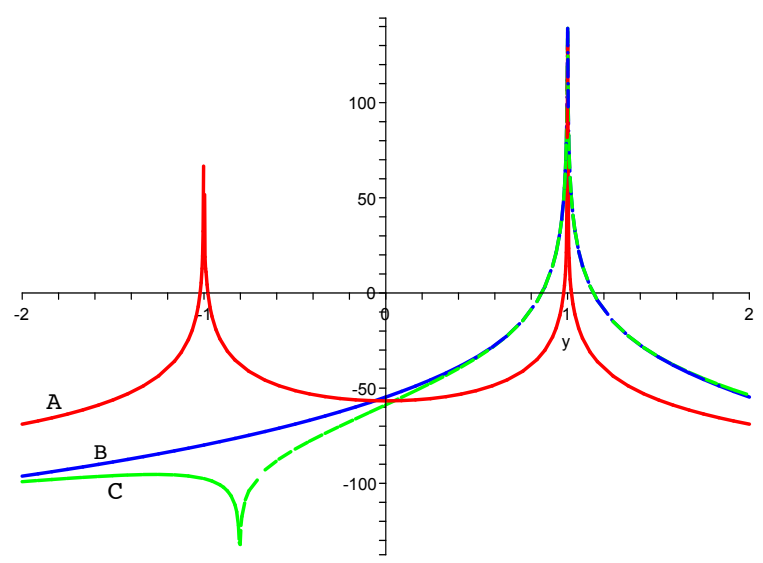

Figure 4. Radial sensitivities of (A) a tripolar cuff electrode, (B) a planar hexagonal patch and (C) a bent hexagonal patch. The vertical axis is in $\mathrm{dB} \mu \mathrm{V}$ and the unit for the horizontal axis is the radius $R$ of the electrode.

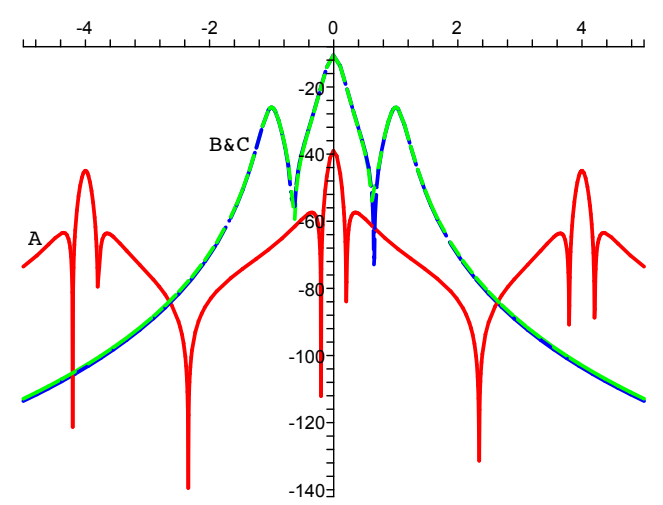

Figure 5. Longitudinal sensitivities on an off-centre (80\% of $\mathrm{R}$ ) axis of (A) a tripolar cuff electrode, (B) a planar hexagonal patch and (C) a bent hexagonal patch. The vertical axis is in $\mathrm{dB} \mu \mathrm{V}$ and the unit for the horizontal axis is the radius $R$ of the electrode.

\section{External sensitivity}

Figure 6 shows the external sensitivities of our three electrodes for an AP placed on the $O x$ or on the $O z$ axis of the electrode. As stated above, the quantity plotted is not a voltage, but is homogeneous to the reciprocal of the cube of
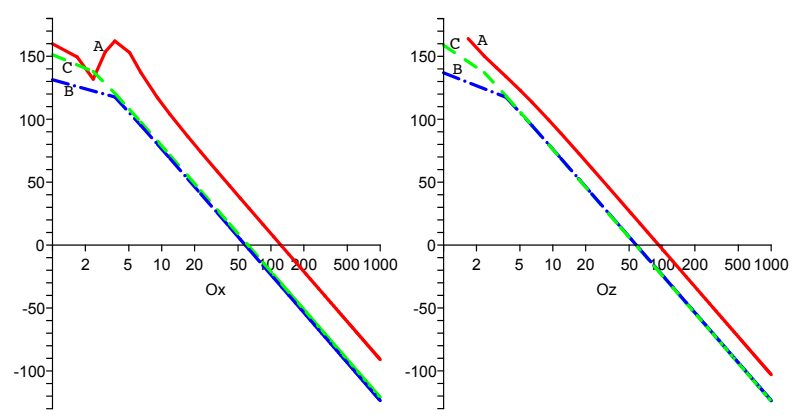

Figure 6. External relative sensitivity along $O x$ and $O z$ axes for (A) a tripolar cuff electrode, (B) a planar hexagonal patch and (C) a bent hexagonal patch. The vertical axis is in $\mathrm{dB}$ and the unit for the horizontal axis is the radius $R$ of the electrode. 
a distance. Nevertheless, we can see on these two graphs that the hexagonal patches exhibit a better rejection of parasitic signals than the tripolar cuff. This improvement is of $32 \mathrm{~dB}$ for $O x$ and $20 \mathrm{~dB}$ for $O z$.

The same study conducted along the $O y$ axis shows that, while the planar patch continues to have the better rejection of parasitic signals, the wrapped hexagonal patch has a sensitivity decreasing slowly along this $O y$ axis. In fact, the bent hexagonal patch only begins to have larger sensitivity than the tripolar cuff for action potentials placed at more than fifty times the radius of the cuff, corresponding to approximately $7 \mathrm{~cm}$. At this distance, the parasitic signal could be neglected in comparison to ENG signal.

\section{Conclusion And Perspectives}

This paper has presented a new architecture of multipolar cuff electrode for efficient ENG signal extraction from nerve. The proposed architecture based on a hexagonal distribution of a large number of poles allows a good rejection of parasitic signal and exhibits a higher sensitivity than the classical tripolar electrode. Moreover, a low noise ASIC with specific low level analog signal processing suitable for this kind of electrode was presented.

The results presented here show that the hexagonal patches allow to have better sensitivity, better spatial selectivity and higher rejection of parasitic signals than the classical tripolar cuff.

To facilitate the signal post-processing on the recording system, we need a maximum of neural data. A tripolar electrode cuff [11] provides only one recording which is the superposition of all action potentials "seen" by the electrode at a given moment. The use of several hexagonal patches on a cuff electrode (see Fig. 1-b) could allow us to record more signals and thus increase the quantity of neural data.

Furthermore, references [4], [12] show that it is possible to extract the direction and the speed of the signal propagation of AP by using several successive poles. This principle is still relevant for our electrode and would thus allow us to obtain more accurate pieces of information about the direction and the speed of AP propagations.

\section{REFERENCES}

[1] M. Djilas, C. Azevedo Coste, K. Yoshida, and G. Cathébras, "Interpretation of ENG signal for FES closed-loop control," in IFESS'06: 11th Annual Conference of the International Functional Electrical Stimulation Society, Miyagi-Zao, Japan, 12-15 Sept. 2006, pp. $85-87$.
[2] A. Ramachandran, J. Sacristan, N. Lago, T. Dörge, M. Osès, K. Koch, and K. Hoffmann, "Multipolar cuff electrodes with integrated pre-amplifier \& filter to interface peripheral nerves for FES application." in 10th Annual Conference of the International FES Society, Montreal, Canada, July 2005. [Online]. Available: http://ifess.org/ifess05/Poster/ $\% 20$ Session $1 \% 201 /$ Ramachandran $1 \% 20$ A.pdf

[3] J. Winter, M. Rahal, N. Taylor, N. Donaldson, and J. Struijk, "Improved spatial filtering of ENG signals using a multielectrode nerve cuff," in 5th Annual Conference of the International Functional Electrical Stimulation Society, Aalborg, Denmark, June 2000. [Online]. Available: http://www.ifess.org/ifess00/Posterl $\% 20$ Session $1 \% 204 /$ Winter.pdf

[4] J. Taylor, N. Donaldson, and J. Winter, "Multipleelectrode nerve cuffs for low-velocity and velocityselective neural recording," Medical and Biological Engineering and Computing, vol. 42, no. 5, pp. 634-643, Sept. 2004.

[5] M. K. Haugland, J. A. Hoffer, and T. Sinkjaer, "Skin contact force information in sensory nerve signals recorded by implanted cuff electrodes," IEEE Transactions on Rehabilitation Engineering, vol. 2, no. 1, pp. 18-28, 1994. [Online]. Available: http: //dx.doi.org/10.1109/86.296346

[6] W. Jensen, T. Sinkjaer, and F. Sepulveda, "Improving signal reliability for on-line joint angle estimation from nerve cuff recordings of muscle afferents," IEEE Transactions on Neural Systems and Rehabilitation Engineering, vol. 10, no. 3, pp. 133-139, Sept. 2002.

[7] L. N. S. Andreasen and J. J. Struijk, "Signal strength versus cuff length in nerve cuff electrode recordings," IEEE Trans. Biomed. Eng., vol. 49, no. 9, pp. 10451050, Sept. 2002.

[8] J. J. Struijk and M. Thomsen, "Tripolar nerve cuff recording: stimulus artifact, EMG and the recorded nerve signal," in Engineering in Medicine and Biology Society, 1995. IEEE 17th Annual Conference, vol. 2, Montreal, Que., Sept. 20-23, 1995, pp. 1105-1106.

[9] C. Pflaum, R. R. Riso, and G. Wiesspeiner, "Performance of alternative amplifier configurations for tripolar nerve cuff recorded ENG," in Engineering in Medicine and Biology Society, 1996. Bridging Disciplines for Biomedicine. Proceedings of the 18th Annual International Conference of the IEEE, vol. 1, Amsterdam, Oct. 31-Nov. 3, 1996, pp. 375-376.

[10] R. B. Stein, Nerve and Muscle. Plenum Press, 1980.

[11] A. Demosthenous and I. F. Triantis, "An adaptive ENG amplifier for tripolar cuff electrodes," IEEE J. Solid-State Circuits, vol. 40, no. 2, pp. 412-421, Feb. 2005.

[12] R. Rieger, M. Schuettler, D. Pal, C. Clarke, P. Langlois, J. Taylor, and N. Donaldson, "Very low-noise ENG amplifier system using CMOS technology," IEEE Transactions on Neural Systems and Rehabilitation Engineering, vol. 14, no. 4, pp. 427-437, Dec. 2006. 